\title{
Kinematics of the Galactic disk from LAMOST Dwarf sample
}

\author{
Yingjie Jing, ${ }^{1}$ Cuihua Du, ${ }^{1 \star}$ Jiayin Gu, ${ }^{2}$ Yunpeng Jia, ${ }^{3}$ Xiyan Peng, ${ }^{3}$ Yuqin Chen, ${ }^{3}$ \\ Zhenyu Wu, ${ }^{3}$ Jun Ma, ${ }^{3} \mathrm{Xu}$ Zhou, ${ }^{3}$ Zihuang Cao, ${ }^{3}$ Yonghui Hou, ${ }^{4}$ Yuefei $\mathrm{Wang}^{4}$ \\ and Yong Zhang 4 \\ ${ }^{1}$ School of Physical Sciences, University of the Chinese Academy of Sciences, Beijing 100049, China \\ ${ }^{2}$ Department of Physics, Wuhan University of Technology, Wuhan 430000, China \\ ${ }^{3}$ Key Laboratory of Optical Astronomy, National Astronomical Observatories, Chinese Academy of Sciences, Beijing 100012, China \\ ${ }^{4}$ Nanjing Institute of Astronomical Optics \& Technology, National Astronomical Observatories, Chinese Academy of Sciences, Nanjing 210042, Ch
}

Accepted XXX. Received YYY; in original form ZZZ

\begin{abstract}
Based on the LAMOST survey and Sloan Digital Sky Survey (SDSS), we use low-resolution spectra of 130,043 F/G-type dwarf stars to study the kinematics and metallicity properties of the Galactic disk. Our study shows that the stars with poorer metallicity and larger vertical distance from Galactic plane tend to have larger eccentricity and velocity dispersion. After separating the sample stars into likely thin-disk and thick-disk sub-sample, we find that there exits a negative gradient of rotation velocity $V_{\phi}$ with metallicity $[\mathrm{Fe} / \mathrm{H}]$ for the likely thin-disk sub-sample, and the thick-disk sub-sample exhibit a larger positive gradient of rotation velocity with metallicity. By comparing with model prediction, we consider the radial migration of stars appears to have influenced on the thin-disk formation. In addition, our results shows that the observed thick-disk stellar orbital eccentricity distribution peaks at low eccentricity $(e \sim 0.2)$ and extends to a high eccentricity $(e \sim 0.8)$. We compare this result with four thick-disk formation simulated models, and it imply that our result is consistent with gas-rich merger model.
\end{abstract}

Key words: Galaxy: disk - Galaxy: formation - Galaxy: kinematics and dynamics - Galaxy: structure

\section{INTRODUCTION}

The formation and evolution of the Galaxy are very important issues in modern astrophysics. Since Gilmore \& Reid (1983) firstly introduced the thick-disk, the basic components of the Galactic disk are thin-disk and thickdisk populations. The two components differ not only in their spatial distribution profiles but also in their kinematics and metallicity (Majewski 1993; Ojha et al. 1996; Freeman \& Bland-Hawthorn 2002). In the spatial distribution, the range of scale height for the thin-disk vary from 220 to $320 \mathrm{pc}$, while that of the thick-disk is from 600 to 1100 pc (Du et al. 2003, 2006; Jia et al. 2014). The range of scale length for the disk (including thin disk and thick disk) vary from 2 to $4 \mathrm{kpc}$, but some evidences for a short scale length for the thick disk were also given by (e.g. Bensby et al.

^ E-mail: ducuihua@ucas.ac.cn
2011; Cheng et al. 2012; Hayden et al. 2015). The thickdisk generally has lower rotational velocity and larger velocity dispersion, has a lower average metallicity $([\mathrm{Fe} / \mathrm{H}]$ $\sim-0.7$ dex)(Gilmore \& Wyse 1985), and has enhanced $\alpha$ element abundances than the thin disk (Gratton et al. 1996; Fuhrmann 1998; Prochaska et al. 2000; Bensby et al. 2003; Reddy et al. 2006; Bensby et al. 2007; Fuhrmann 2008).

The origin of the thick disk have been investigate by authors (e.g. Quinn \& Goodman 1986; Freeman 1987; Abadi et al. 2003; Brook et al. 2004, 2005, 2007; Schönrich \& Binney 2009) and have not been resolved. Some currently discussed models of formation mechanisms for the thick disk predict various trend between the kinematics properties and metallicity of disk stars, as well as between their kinematics and spatial distributions. For example, models of Gas-rich merger predict a rotational velocity gradient with Galactocentric distance for disks stars near the solar radius (Brook et al. 2007). Models of disk heating 
via satellite mergers or a growing thin disk can induce a notable increase in the mean rotation and velocity dispersions of thick disk stars (Villalobos et al. 2010). Sales et al. (2009) also showed that the distribution of orbital eccentricities for nearby thick disk stars could provide constraints on these proposed formation models. Comparisons the predictions of the models with observed kinematics properties of Galactic disk are helpful to understand the formation and evolution of Galaxy.

To understanding the formation and chemical evolution of the Galaxy components, we need more chemical and kinematics information of a large number of stars in larger areas which will greatly increase the spatial coverage of the Galaxy. The large-scale spectroscopic surveys make it possible by providing ideal databases such as radial velocities and stellar atmospheric parameters (Teff, $\log g,[\mathrm{Fe} / \mathrm{H}]$, etc). A number of papers have employed the kinematics characters and chemical abundances to study the Galactic structure and formation, based on spectroscopic survey data. For instance, Bond et al. (2010), Carollo et al. (2010) , Lee et al. (2011) and Smith et al. (2009) have characterized the halo and disk base on Sloan Digital Sky Survey (SDSS; York et al. 2000) and its subsurvey Sloan Extension for Galactic Understanding and Exploration (SEGUE; Aihara et al. 2011; Yanny et al. 2009; Beers et al. 2006). SDSS III's Apache Point Observatory Galactic Evolution Experiment (APOGEE; Eisenstein et al. 2011) has higher resolution than SEGUE, also being used to explore the kinematic of disk (e.g. Bovy et al. 2012, 2015; Ness et al. 2016). Comparing to SEGUE, the Radial Velocity Experiment (RAVE) provide a bright complement to the SEGUE sample (Siebert et al. 2011). Many works on kinematics of Galactic disk (e.g. Binney et al. 2014; Casetti-Dinescu et al. 2011; Siebert et al. 2008) have been done on RAVE data. Of course, there also have some works based on Gaia-ESO internal data-release (e.g. Recio-Blanco et al. 2014; Kordopatis et al. 2015). The ongoing Large Sky Area Multi-Object Fiber Spectroscopic Telescope survey (LAMOST, also called Guoshoujing Telescope; Cui et al. 2012; Deng et al. 2012; Zhao et al. 2012; Luo et al. 2012) has release more than two millions stellar spectra with stellar parameters in the DR2 catalog. This data set will provide a vast resource to study details of the velocity distribution and give constraints on the dynamical structure and evolution of the Galactic disk.

In this study, we make use of the F/G dwarf stars selected from the LAMOST survey to explore the observed correlations of kinematic velocity and orbital eccentricity with metallicity and distance from the Galactic plane. We also compare the observation results with the predictions of different models and expect to obtain some clues for the disk formation. The outlines of this paper are as follows. In Section 2, we take a brief overview of the LAMOST, observation data and derive the individual three-dimensions velocity. Section 3 presents the results of the observation. The discussions are given in Section 4. A summary and conclusions are given in Section 5.

\section{THE DATA}

\subsection{Observational Data}

The Large Sky Area Multi-object Fiber Spectroscopic Telescope (LAMOST) survey is a 4 meter quasi-meridian reflective Schmidt telescope at the Xinglong Station of the National Astronomical Observatories (NAOC), Chinese Academy of Sciences. The field of view of the CCD is $5^{\circ}$. It is a powerful instrument to survey the sky with the capability of recording 4,000 spectra simultaneously. The spectrograph of LAMOST has a resolution of $\mathrm{R} \sim 1,800$ and range span-

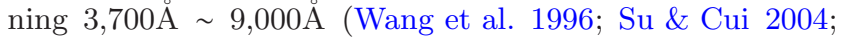
Cui et al. 2012). LAMOST has completed 3 years of survey operations plus a Pilot Survey, and internally released a total $\sim 5.7$ million spectra to the collaboration. Of these, $\sim 3.7$ million are AFGK-type stars with estimated stellar atmospheric parameters as well as radial velocities. Stellar atmospheric parameters (including spectral types, $[\mathrm{Fe} / \mathrm{H}], \log g$ ) are derived by Ulyss software (Wu et al. 2011). The radial velocities are measured by cross-correlation between spectra and template spectra from the Elodie library (Moultaka et al. 2004). The survey reaches a limiting magnitude of $r=17.8$ (Where $r$ denotes magnitude in the SDSS $r$-band), and most targets is brighter than $r \sim 17$.

In this study, we use stellar atmospheric parameters (including spectral types, $[\mathrm{Fe} / \mathrm{H}], \log g$ ) and radial velocity from LAMOST DR2 catalogs. Our initial sample was obtained by a cross referencing between the LAMOST DR2 and SDSS DR12 photometric catalog based on stellar position. The proper motions of the sample are obtained by cross-identifying with SDSS-POSS proper-motion catalog (Munn et al. 2004, 2008). Stars without SDSS photometry and proper motions were eliminated to estimate photometric distances by SDSS color. Next, we select F/G dwarf stars $(\log g>3.5)$ with $\mathrm{S} / \mathrm{N}>15$ at $g$ band. In order to use the photometric parallax relation, we restrict sample at $0.2<g-i<4.0$. This cut reduces the numbers of stars to 180,759 .

In order to investigate the systematics of the radial velocities, we cross identify the LAMOST data (S/N > 20 at $g$ band and $\log g>3.5$ ) with the SDSS-SSPP data (average $\mathrm{S} / \mathrm{N}>20$ ) and select 3077 common stars as a sample with good parameter estimates. As shown in Figure 1, the offset of radial velocities between the LAMOST pipeline and the SDSS-SSPP is concentrate on $-6.76 \mathrm{~km} \mathrm{~s}^{-1}$ with a dispersion of $7.9 \mathrm{~km} \mathrm{~s}^{-1}$. Tian et al. (2015) also reports that the radial velocity derived from the LAMOST pipeline is slower by $-5.7 \mathrm{~km} \mathrm{~s}^{-1}$ compared with APOGEE. The reason for this offset is unclear. In this study, in order to match the other survey data, we add an additional $6.76 \mathrm{~km} \mathrm{~s}^{-1}$ to the derived LAMOST radial velocity.

\subsection{The photometric parallax and spatial velocity}

We estimate the distance using a photometric parallax method described by Ivezić et al. (2008), which gives the absolute magnitude in the $r$ band as a function of $g-i$ and $[\mathrm{Fe} / \mathrm{H}]$. We then derive the $U, V, W$ space velocity components from the distances, radical velocities and proper motions. Here, we apply $\left(U^{\odot, \text { pec }}, V^{\odot, \text { pec }}, W^{\odot, \text { pec }}\right)=(-11.1,-12.24,7.25) \mathrm{km}$ $\mathrm{s}^{-1}$ (Schönrich et al. 2010) to adjust for the solar peculiar 


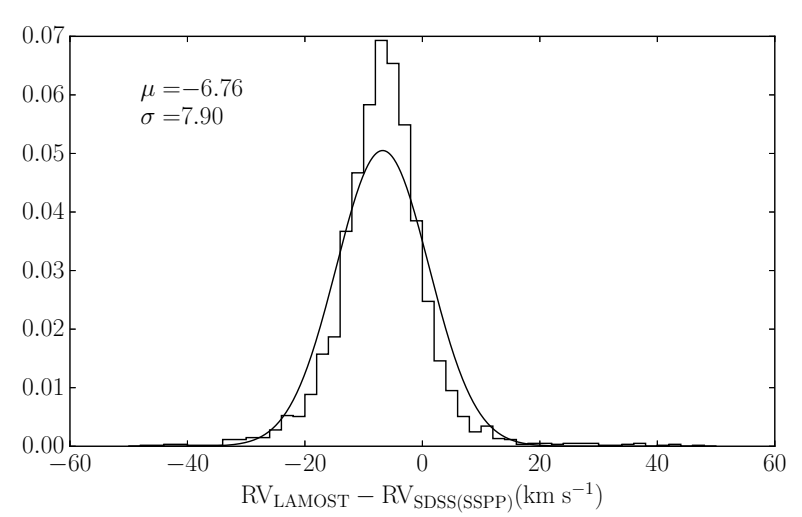

Figure 1. The offsets of the radial velocities between the LAMOST pipeline and the SEGUE Stellar Parameter Pipeline. The histogram is the offset distribution of 3077 common stars, and the solid curve is Gaussian fit to the offset distribution.

motions with respect to the local standard of rest (LSR). In order to obtain the Galactocentric cylindrical velocity components, we adopt $V_{\text {LSR }}=220 \mathrm{~km} \mathrm{~s}^{-1}$ (Gunn et al. 1979; Feast \& Whitelock 1997) and $R_{\odot}=8 \mathrm{kpc}$.

We select these stars with $6.5<R<9.5 \mathrm{kpc}$ and $0.1<|\mathrm{Z}|<3 \mathrm{kpc}$, this cut reduces the sample stars to 130,043 . The reasons for the selection criteria $|Z|>0.1 \mathrm{kpc}$ are that these stars are not well observed in the LAMOST survey and SDSS survey because most of them are too bright (Tian et al. 2015) and to avoid the effects of overestimated interstellar extinction correction for the these stars (Jurić et al. 2008). The median estimated errors on the $V_{\phi}, V_{R}$ and $V_{Z}$ of the sample stars are 16,16 and $17 \mathrm{~km} \mathrm{~s}^{-1}$, respectively. The spatial distribution of the sample in the cylindrical Galactic coordinates $\mathrm{Z}-R$ panel is plotted in Figure 2. Figure 3 gives the iso-density contours of the velocity components $U, V$ and $W$ of the sample stars with metallicity, and the filled circles denote the mean value of velocity components in different metallicity interval. As shown in Figure 3 , there is no significant association with $[\mathrm{Fe} / \mathrm{H}]$ for $U$ and $W$, however, for $V$ which is associate with $V_{\phi}$, there has a relative small-scale spatial distribution. Summarizing the criteria adopted in our sample selection, the sample stars satisfy $6.5<R<9.5 \mathrm{kpc}, 0.1<|Z|<3 \mathrm{kpc}$, log $g>3.5$, $-1.2<[\mathrm{Fe} / \mathrm{H}]<0.6$. It shows that our sample stars could be mainly contributed from the disk system. In total, there are 130,043 sample stars in this study.

\section{RESULTS OF THE OBSERVATIONS}

\subsection{Correlation of orbital eccentricities with metallicity}

We investigate the orbital properties of our sample stars by adopting the Galaxy potential model of Paczynski (1990) proposed by Miyamoto \& Nagai (1975). The model is axissymmetric and fully analytic, and consists of a spherical central bulge, a highly flattened disk and a nearly spherical halo. We derive the orbital eccentricity $e$, defined as $e=\left(r_{\text {apo }}-r_{\text {peri }}\right) /\left(r_{\text {apo }}+r_{\text {peri }}\right)$, where $r_{\text {peri }}$ denote the closest approach of an orbit to the Galactic center (i.e., the perigalactic distance), and $r_{\text {apo }}$ denote the farthest extent of an orbit

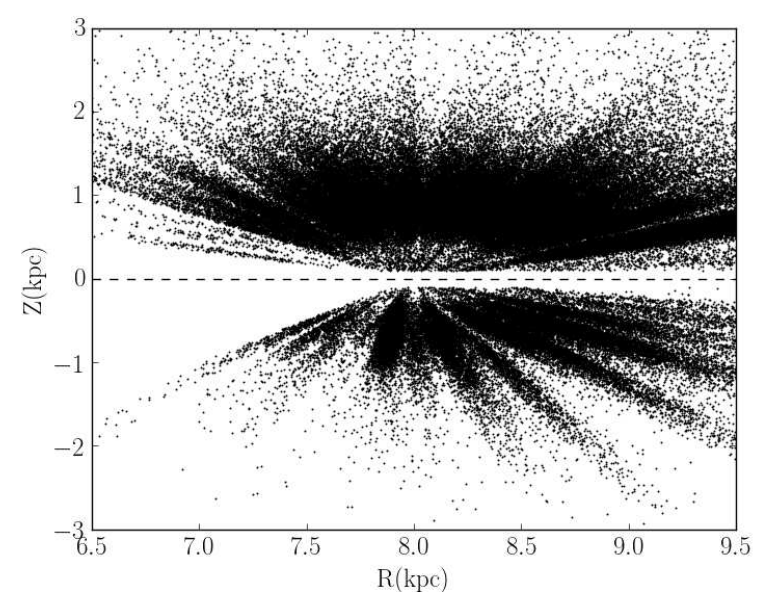

Figure 2. Spatial distribution in the the cylindrical Galactic coordinates $Z-R$ plane of the 130,043 sample stars in this study. The dashed line represents the Galactic plane.

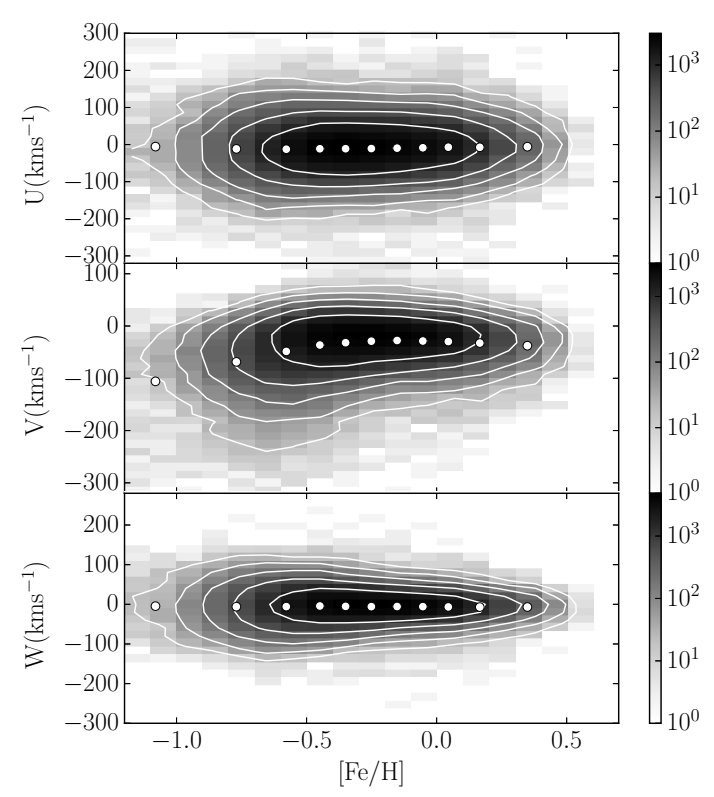

Figure 3. The iso-density contours of $U, V$ and $W$ distribution with metallicity $[\mathrm{Fe} / \mathrm{H}]$, respectively. The filled circles denote the mean value of $U, V$ and $W$ in different metallicity intervals.

from the Galactic center (i.e., the apogalactic distance). We performed a Monte Carlo simulation to estimate the errors of orbital eccentricities: for each star, we computed 1000 times of the orbital eccentricities by changing the Galactic coordinates and the velocities every time, assuming Gaussian distributions around their values and a dispersion according to their estimated errors. From these 1000 orbits we computed the standard deviation for each eccentricity. The errors of orbital eccentricity are smaller than 0.15 dex for most stars. Figure 4 shows the eccentricity versus metallicity $[\mathrm{Fe} / \mathrm{H}]$ for the sample stars in this study. The eccentricity and metallicity of our sample have a wide dispersion. But most of the stars have an eccentricity $<0.6$. 


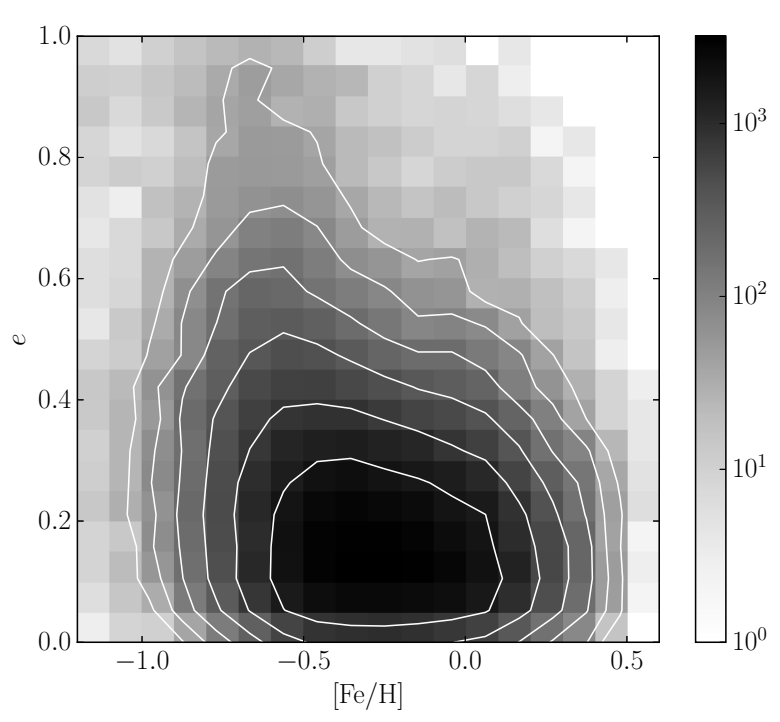

Figure 4. Metallicity $[\mathrm{Fe} / \mathrm{H}]$ vs. orbital eccentricity for the sample stars.

We compute the mean orbital eccentricity in different metallicity and $|Z|$ distance interval and the results are indicated in Figure 5. The bin of $[\mathrm{Fe} / \mathrm{H}]$ is 0.2 dex and the filled circles, open circles and filled squares denote the stars at $0.1<|Z|<0.5 \mathrm{kpc}, 0.5<|Z|<1 \mathrm{kpc}$, and $1<|Z|<3$ $\mathrm{kpc}$, respectively. As shown in this figure, the eccentricity increases with increasing distance from the Galactic plane and the change trend of eccentricity versus $[\mathrm{Fe} / \mathrm{H}]$ is different at $-1.2<[\mathrm{Fe} / \mathrm{H}]<-0.5$ and $-0.2<[\mathrm{Fe} / \mathrm{H}]<0.6$. In the first metallicity range, the sample is dominated by the thick-disk population, the trend of the orbital eccentricity generally decreases as the metallicity increases, and there is a relatively larger gradient with increasing metallicity. In the second metallicity range which is dominated by thin-disk population, there is an almost flat trend of orbital eccentricity with metallicity $[\mathrm{Fe} / \mathrm{H}]$ (the gradient approach zero except at $1<|Z|<3 \mathrm{kpc}$ ), and indicate that the orbital eccentricity is independent of metallicity. These results agree well with the recent determinations from the SEGUE data (Lee et al. 2011) which also found the thick-disk stars exhibit a strong trend of eccentricity with metallicity (about $-0.2 \mathrm{dex}^{-1}$ ), while the eccentricity does not change with metallicity for the thin-disk sub-sample. However, for those stars in distance interval $1<|Z|<3 \mathrm{kpc}$ and the second metallicity range $(-0.2<[\mathrm{Fe} / \mathrm{H}]<0.6)$, the eccentricity has a little increase with increasing metallicity. We consider that these stars are assimilated into the thick disk from orbits near the Galactic disk plane or radial migration have influenced the structural and chemical evolution of the Galactic disk. Even, it is possible that these high eccentric orbit and metalrich stars origin from bulge, Pompéia et al. (2002) show that there exist bulge-like stars with rich metallicities and very eccentric orbits $(e>0.25)$. But their sample stars have nearly distance from the Galactic plane $(|Z|<1)$.

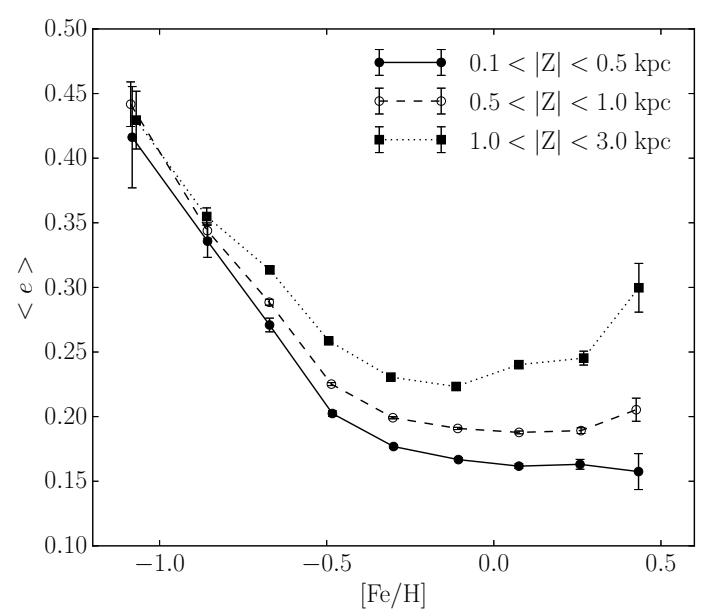

Figure 5. The orbital eccentricity as a function of $[\mathrm{Fe} / \mathrm{H}]$ for the selected sample. The filled circles, open circles and filled squares correspond the stars at $0.1<|Z|<0.5 \mathrm{kpc}, 0.5<|Z|<1 \mathrm{kpc}$, and $1<|Z|<3 \mathrm{kpc}$, respectively. The bin represent a range of $0.2 \mathrm{dex}$ in metallicity.

\subsection{The properties of rotational velocity}

In this section, we use our sample to examine the properties of the rotational velocity with metallicity $[\mathrm{Fe} / \mathrm{H}], R$ and $|Z|$. Figure 6 shows the mean rotational velocity distribution as a function of metallicity $[\mathrm{Fe} / \mathrm{H}]$ for the selected sample. The metallicity interval and the value in each interval are also listed in Table 1. As shown in Figure 6, the mean rotational velocity increases with increased metallicity and has a peak at $[\mathrm{Fe} / \mathrm{H}] \sim-0.2$, then it becomes a slightly decrease when $[\mathrm{Fe} / \mathrm{H}]>-0.2$. A plausible explanation is that highmetallicity range is dominated by thin-disk population and the rotational velocity of thin-disk decreases with increased metallicity $[\mathrm{Fe} / \mathrm{H}]$, however most stars at lower metallicity belong to thick disk population and the rotational velocity of thick-disk increases with increasing metallicity $[\mathrm{Fe} / \mathrm{H}]$. In order to check this explanation, we select the likely thindisk and thick-disk sub-sample from our sample following the prescription of Bensby et al. (2003, 2014). We calculate the likelihood each star belongs to the thin-disk, the thickdisk and the halo assuming the space velocities of three components are distributed as Gaussian. The adopted value of the local stellar densities, velocity dispersions in $U, V$, and $W$, and the asymmetric drifts here are the same as that in Bensby et al. (2014). We select the likely thick-disk stars as those with $-1.2<[\mathrm{Fe} / \mathrm{H}]<-0.3$, relative likelihood for the thick-disk to thin-disk (TD/D) $>5$ and thick-disk to halo $(\mathrm{TD} / \mathrm{H})>2$, the likely thin-disk stars as those with $[\mathrm{Fe} / \mathrm{H}]$ $>-0.5$ and $\mathrm{TD} / \mathrm{D}<0.2$.

Figure 7 shows the result of this verification. The values of each circle is obtained by passing a box of 500 stars, with an overlap of 100 stars per bin through the data. The slopes are obtained by performing least-square fits to each unbinned sub-sample. The uncertainties of the slopes are calculated by resampling each sub-sample with replacement 1000 times, assuming Gaussian distributions around values of $[\mathrm{Fe} / \mathrm{H}]$ and $V_{\phi}$ and a dispersion according to their estimated errors. As shown in Figure 7, there exist a clear gradient of rotational velocity with metallicity $[\mathrm{Fe} / \mathrm{H}]$ for both 


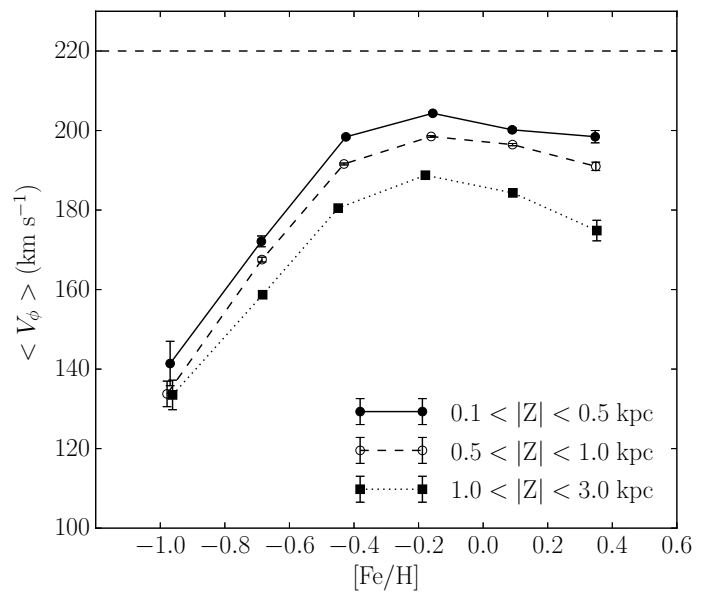

Figure 6. Distribution of the mean rotational velocity as a function of metallicity $[\mathrm{Fe} / \mathrm{H}]$ for the selected sample with different $|\mathrm{Z}|$ intervals. The filled circles, open circles and filled squares denote the stars at $0.1<|Z|<0.5 \mathrm{kpc}, 0.5<|Z|<1 \mathrm{kpc}$, and $1<|Z|<$ $3 \mathrm{kpc}$, respectively. The dashed line is the adopted $V_{\mathrm{LSR}}=220$ $\mathrm{km} \mathrm{s}^{-1}$ for the motion of the local standard of rest.

the likely thin-disk and the likely thick-disk sub-sample at different distance from the Galactic plane. A strong gradient of about 28.1 to $39.6 \mathrm{~km} \mathrm{~s}^{-1} \mathrm{dex}^{-1}$ for the likely thick-disk sub-sample and a clear gradient of about -6.5 to $-9.5 \mathrm{~km}$ $\mathrm{s}^{-1} \mathrm{dex}^{-1}$ for the likely thin-disk sub-sample are obtained in the different distance slice $|Z|$. Lee et al. (2011) also reported similar gradients for their likely thin- and thick-disk sub-sample separated base on $[\alpha / \mathrm{Fe}]$ ratio versus $[\mathrm{Fe} / \mathrm{H}]$ derived from spectra of G-type dwarfs from the SEGUE survey. The gradient for the thick-disk population agrees with the claim of Spagna et al. (2010), who derived a similar gradient of 40 to $50 \mathrm{~km} \mathrm{~s}^{-1} \mathrm{dex}^{-1}$, amongst their thick disk stars located between 1 and $3 \mathrm{kpc}$ from the Galactic plane and with metallicity $-1.0<[\mathrm{Fe} / \mathrm{H}]<-0.5$.

Moreover, Figure 6 also indicates the rotational velocity becomes slow with increased distance from Galactic plane $|Z|$. In order to explore this behavior more detailed, we select stars in the high-metallicity range $[\mathrm{Fe} / \mathrm{H}]>-0.1$ and intermediate-metallicity range $-0.8<[\mathrm{Fe} / \mathrm{H}]<-0.6$, and derive the mean rotational velocity as a function of $|Z|$ and $R$. We derive the mean rotation velocity by passing a box of 1000 stars, with an overlap of 600 stars per bin through the data. The results are given in Figure 8. We obtain the gradients of mean rotational velocity $V_{\phi}$ with distance from the Galactic plane are $-18.5 \mathrm{~km} \mathrm{~s}^{-1} \mathrm{kpc}^{-1}$ and $-14.2 \mathrm{~km} \mathrm{~s}^{-1} \mathrm{kpc}^{-1}$ for the high-metallicity and intermediate-metallicity sub-sample, respectively. The gradient of intermediate-metallicity sub-sample is smaller than that obtained by Carollo et al. (2010), $-36 \mathrm{~km} \mathrm{~s}^{-1} \mathrm{kpc}^{-1}$, base on same metallicity range $-0.8<[\mathrm{Fe} / \mathrm{H}]<-0.6$ at $0<|\mathrm{Z}|<$ $4 \mathrm{kpc}$, and larger than that obtained by Lee et al. (2011), $-9.4 \mathrm{~km} \mathrm{~s}^{-1} \mathrm{kpc}^{-1}$ for thick-disk stars. The bottom panel of Figure 8 indicates only a negligible rotational velocity gradient with the Galactocentric radius $R$ for both sub-samples (only $2.4 \mathrm{~km} \mathrm{~s}^{-1} \mathrm{kpc}^{-1}$ and $1.6 \mathrm{~km} \mathrm{~s}^{-1} \mathrm{kpc}^{-1}$ ), which is consistent with a flat rotation curve in the solar neighborhood.

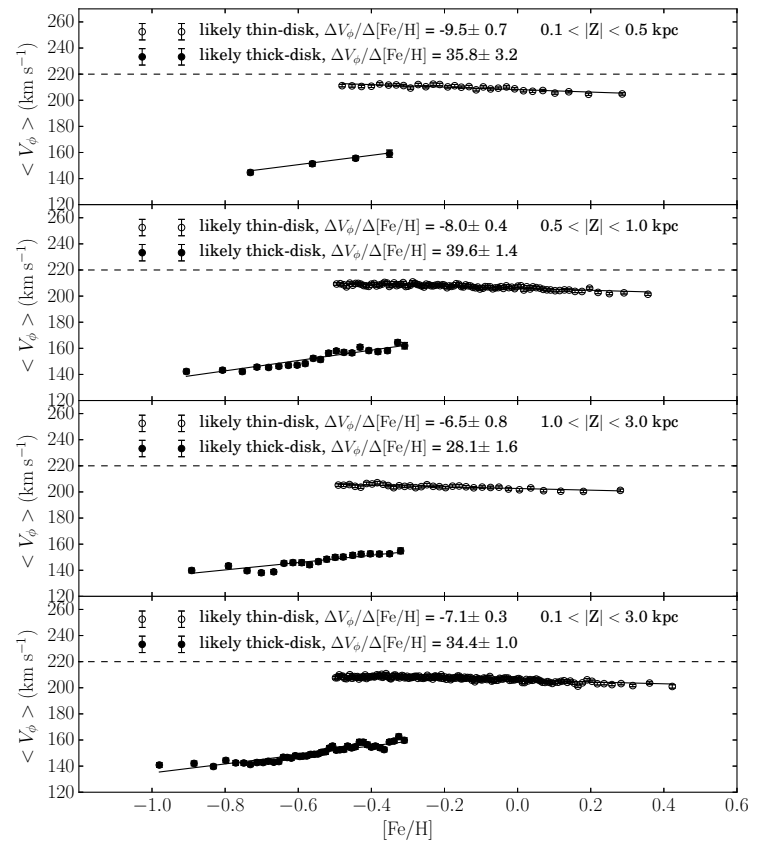

Figure 7. Rotation velocity gradients with metallicity $[\mathrm{Fe} / \mathrm{H}]$ for different intervals in distance from the Galactic plane, for stars assigned to likely thin-disk (open circles) and thick-disk (filled circles) populations.

Table 1. Rotational Properties of the Selected Sample

\begin{tabular}{|c|c|c|c|}
\hline $\begin{array}{c}{[\mathrm{Fe} / \mathrm{H}]} \\
(\mathrm{dex})\end{array}$ & $\mathrm{N}_{\text {Stars }}$ & $\begin{array}{c}\left\langle V_{\phi}\right\rangle \\
\left(\mathrm{km} \mathrm{s}^{-1}\right)\end{array}$ & $\begin{array}{c}\sigma_{V_{\phi}} \\
\left(\mathrm{km} \mathrm{s} \mathrm{s}^{-1}\right)\end{array}$ \\
\hline \multicolumn{4}{|c|}{$0.1<|Z|<0.5 \mathrm{kpc}$} \\
\hline-1.2 to -0.9 & 142 & $141.4 \pm 5.6$ & $66.4 \pm 4.0$ \\
\hline-0.9 to -0.6 & 1392 & $172.1 \pm 1.3$ & $50.7 \pm 1.0$ \\
\hline-0.6 to -0.3 & 6285 & $198.4 \pm 0.5$ & $36.1 \pm 0.3$ \\
\hline-0.3 to 0 & 7702 & $204.3 \pm 0.3$ & $29.1 \pm 0.2$ \\
\hline 0 to 0.3 & 3478 & $200.2 \pm 0.5$ & $27.9 \pm 0.3$ \\
\hline 0.3 to 0.6 & 275 & $198.5 \pm 1.5$ & $25.6 \pm 1.1$ \\
\hline \multicolumn{4}{|c|}{$0.5<|Z|<1.0 \mathrm{kpc}$} \\
\hline-1.2 to -0.9 & 565 & $133.8 \pm 3.2$ & $76.2 \pm 2.3$ \\
\hline-0.9 to -0.6 & 7055 & $167.5 \pm 0.6$ & $50.6 \pm 0.4$ \\
\hline-0.6 to -0.3 & 25526 & $191.6 \pm 0.2$ & $39.4 \pm 0.2$ \\
\hline-0.3 to 0 & 26643 & $198.5 \pm 0.2$ & $32.0 \pm 0.2$ \\
\hline 0 to 0.3 & 11566 & $196.5 \pm 0.3$ & $30.3 \pm 0.2$ \\
\hline 0.3 to 0.6 & 911 & $191.0 \pm 1.1$ & $31.8 \pm 0.7$ \\
\hline \multicolumn{4}{|c|}{$1.0<|Z|<3.0 \mathrm{kpc}$} \\
\hline-1.2 to -0.9 & 439 & $133.4 \pm 3.7$ & $77.7 \pm 2.6$ \\
\hline-0.9 to -0.6 & 6059 & $158.7 \pm 0.7$ & $50.1 \pm 0.5$ \\
\hline-0.6 to -0.3 & 16759 & $180.5 \pm 0.4$ & $47.7 \pm 0.3$ \\
\hline-0.3 to 0 & 11023 & $188.8 \pm 0.4$ & $40.9 \pm 0.5$ \\
\hline 0 to 0.3 & 3840 & $184.3 \pm 0.7$ & $44.3 \pm 0.5$ \\
\hline 0.3 to 0.6 & 383 & $174.8 \pm 2.6$ & $50.5 \pm 1.8$ \\
\hline
\end{tabular}



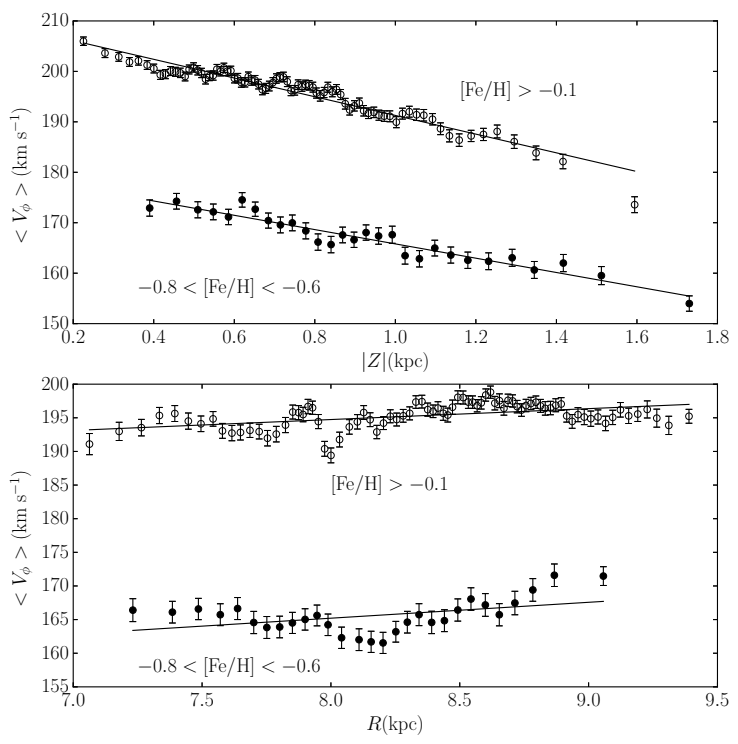

Figure 8. The mean rotational velocity with distance from the Galactic plane $|Z|$ (top panel) and with Galactocentric radius $R$ (bottom panel) for two different metallicity intervals.

\subsection{Correlation of velocity dispersion with metallicity and $|Z|$}

In this section, we investigate the correlation of velocity dispersion with metallicity and vertical distance from the Galactic plane. Figure 9 shows the observed velocity dispersion $\left(\sigma_{U}, \sigma_{V}, \sigma_{W}\left(\sigma_{Z}\right), \sigma_{R}, \sigma_{\phi}\right)$ as a function of metallicity $[\mathrm{Fe} / \mathrm{H}]$ at different $|\mathrm{Z}|$ intervals. We first examine the properties of $\sigma_{U}, \sigma_{V}$ and $\sigma_{W}$. As shown in Figure 9, the behavior of the velocity dispersion $\sigma_{U}, \sigma_{V}$ and $\sigma_{W}$ versus metallicity $[\mathrm{Fe} / \mathrm{H}]$ is particularly different at $-1.2<[\mathrm{Fe} / \mathrm{H}]<-0.2$ and $[\mathrm{Fe} / \mathrm{H}]>-0.2$. In the first metallicity interval, the velocity dispersions have a relatively larger negative gradient with increased metallicity $[\mathrm{Fe} / \mathrm{H}]$ in any distance $|Z|$. Especially for $[\mathrm{Fe} / \mathrm{H}]<-0.6$, the trends of the velocity dispersion with metallicity for the three velocity components are consistent with the results from 1203 solar-neighborhood metal-poor stars with $[\mathrm{Fe} / \mathrm{H}]<-0.6$ in Chiba \& Beers (2000). In the second metallicity range, most stars belong to the thin-disk population, the gradient is negligible at $0.1<|Z|<0.5 \mathrm{kpc}$ and $0.5<|Z|<1 \mathrm{kpc}$, while the velocity dispersions $\sigma_{U}$ and $\sigma_{V}$ begin to increase with increased metallicity $[\mathrm{Fe} / \mathrm{H}]$ at 1 $<|\mathrm{Z}|<3 \mathrm{kpc}$. The trend for metal-rich star at $1<|\mathrm{Z}|<3$ $\mathrm{kpc}$ is similar with Figure 5, which reveal those stars possibly belong to thick-disk population because of the larger velocity dispersion and eccentricity. We also notice that the behaviors of velocity dispersions $\sigma_{R}$, and $\sigma_{\phi}$ resemble $\sigma_{U}$, and $\sigma_{\mathrm{V}}$ with metallicity $[\mathrm{Fe} / \mathrm{H}]$ and $|Z|$. This is a natural consequence of our sample far away from Galactic center and having relative small-scale spatial distribution.

In addition, Figure 9 clearly show an increase of velocity dispersions with increased $|Z|$, especially for the metal-rich stars. We derive the gradient of dispersions with distance $|Z|$ at metallicity ranges $-1.2<[\mathrm{Fe} / \mathrm{H}]<-0.6,-0.6<[\mathrm{Fe} / \mathrm{H}]$ $<-0.1$, and $-0.1<[\mathrm{Fe} / \mathrm{H}]<0.6$. The values are also listed in Table 2. The gradients are obtained by performing least- square fits to the value of dispersions which are derived by passing a box of 800 stars, with an overlap of 300 stars per bin, through the data of each velocity component. As shown in Table 2, metal-rich stars have larger gradient of dispersions with $Z$. In metallicity range $-1.2<[\mathrm{Fe} / \mathrm{H}]<-0.6$, most stars belong to the thick-disk population and shows a smaller gradient $\left(5.7,5.4\right.$, and $\left.1.6 \mathrm{~km} \mathrm{~s}^{-1} \mathrm{kpc}^{-1}\right)$ of $\sigma_{U}, \sigma_{V}$, and $\sigma_{W}$ with distance $|Z|$. In contrast, Moni Bidin et al. (2010) derived a vertical gradient of $\sigma_{U}, \sigma_{V}$, and $\sigma_{W}$ of $6.2,4.5$, and $2.8 \mathrm{~km} \mathrm{~s}^{-1} \mathrm{kpc}^{-1}$ for thick-disk stars at $2<|Z|<4.5 \mathrm{kpc}$, and Moni Bidin et al. (2012) also derived a similar gradient of $6.3,4.1$, and $2.4 \mathrm{~km} \mathrm{~s}^{-1} \mathrm{kpc}^{-1}$ for stars at $1.5<|Z|<4.5 \mathrm{kpc}$.

\section{DISCUSSIONS}

\subsection{Correlations between Rotational Velocity and Metallicity}

The radial migration models (Sellwood \& Binney 2002; Roškar et al. 2008b; Schönrich \& Binney 2009; Sales et al. 2009; Minchev \& Famaey 2010) suggest that the energy and angular momentum changes occur from interactions with transient spiral arms, which move stars at the corotation resonance inward or outward in radius while preserving their nearly circular orbits (Loebman et al. 2011). Loebman et al. (2011) employed a $N$-body simulation with radial migration and found a gradient of about $-24.8 \mathrm{~km} \mathrm{~s}^{-1} \mathrm{dex}^{-1}$ with $[\mathrm{Fe} / \mathrm{H}]$ for the rotational velocities of their younger stars (identified with thin-disk component), the gradient was more larger when restricting the metallicity with $-0.2<[\mathrm{Fe} / \mathrm{H}]<0.4$. The result is qualitative agreements with our likely thindisk component (identified by kinematic criteria). Loebman et al. (2011) did't find significant gradient (only $1.4 \mathrm{~km}$ $\mathrm{s}^{-1} \mathrm{dex}^{-1}$ ) for their older stars $(>7 \mathrm{Gyr}$, which generally matched the observed properties of the thick-disk component). This result is disagreement with our observed large positive gradient of rotational velocity with $[\mathrm{Fe} / \mathrm{H}]$ for likely thick-disk component. However, the pure N-body models of Curir et al. (2012) predicted a lager positive slope $(\sim 60 \mathrm{~km}$ $\left.\mathrm{s}^{-1} \mathrm{dex}^{-1}\right)$ of $V_{\phi}$ with $[\mathrm{Fe} / \mathrm{H}]$ for their thick-disk population which are at $1.5 \mathrm{kpc}<|\mathrm{Z}|<2.0 \mathrm{kpc}$ and persist up to 6 Gyr. Their simulation contained radial migration and heating processes of stars from the inner region of the disk and assumed an initial radial chemical gradient as that suggested by Spitoni \& Matteucci (2011). As a result, the correlation between $V_{\phi}$ and metallicity $[\mathrm{Fe} / \mathrm{H}]$ reveal that the radial migration appears to play an important role in the formation of thin-disk population, but it can't reach a firm conclusion about the effect of radial migration in the formation of thick-disk population.

\subsection{Distribution of Orbital Eccentricities of Thick-Disk Stars}

Sales et al. (2009) demonstrated that the orbital eccentricity distribution of nearby thick-disk stars may help elucidate the dominant formation mechanism of the thick-disk and they also show the distribution for different thick-disk formation models. In this section, we investigate the eccentricity distribution of observed thick-disk stars and compare the result with their simulated result. 


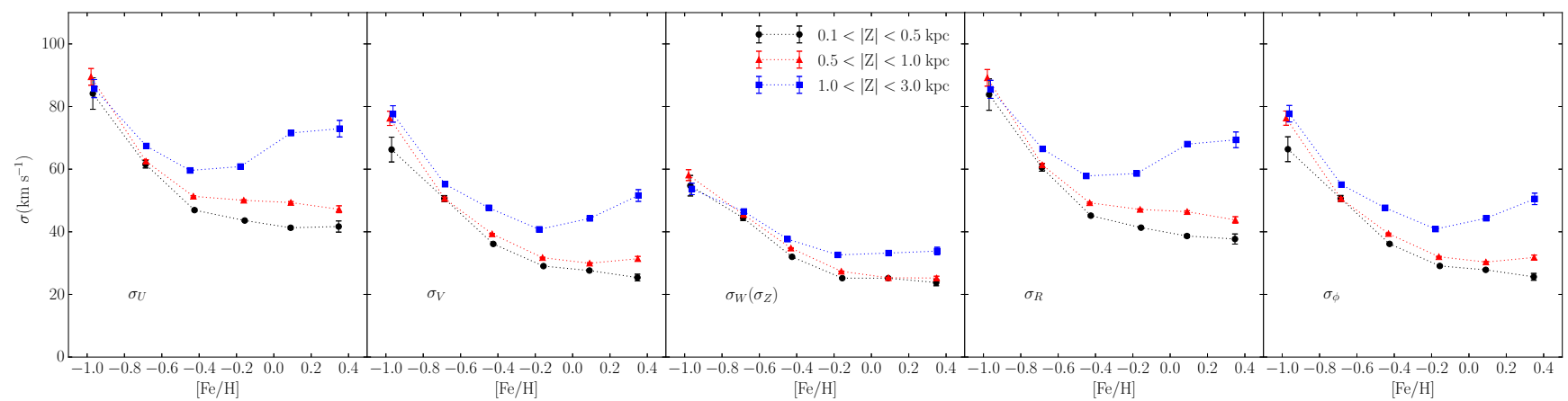

Figure 9. Distribution of the velocity dispersion $\left(\sigma_{U}, \sigma_{V}, \sigma_{W}\left(\sigma_{Z}\right), \sigma_{R}, \sigma_{\phi}\right)$ vs. [Fe/H] for the star in different $|Z|$ intervals. The filled circles, triangles and squares denote the stars at $0.1<|\mathrm{Z}|<0.5 \mathrm{kpc}, 0.5<|\mathrm{Z}|<1 \mathrm{kpc}$, and $1<|\mathrm{Z}|<3 \mathrm{kpc}$, respectively. The bins in metallicity are same with Table 1.

Table 2. The Gradients of Velocity Dispersions with $|Z|$

\begin{tabular}{cccccc}
\hline \hline $\begin{array}{c}{[\mathrm{Fe} / \mathrm{H}]} \\
\text { (dex) }\end{array}$ & $\partial \sigma_{U} / \partial Z$ & $\partial \sigma_{V} / \partial Z$ & $\begin{array}{c}\partial \sigma_{\mathrm{W}} / \partial Z\left(\partial \sigma_{Z} / \partial Z\right) \\
\left(\mathrm{km} \mathrm{s}^{-1} \mathrm{kpc}^{-1}\right)\end{array}$ & $\partial \sigma_{R} / \partial Z$ & $\partial \sigma_{\phi} / \partial Z$ \\
\cline { 3 - 6 } & & & $1.6 \pm 0.5$ & $5.9 \pm 0.8$ & $5.3 \pm 0.7$ \\
\hline-1.2 to -0.6 & $5.7 \pm 0.8$ & $5.4 \pm 0.7$ & $7.2 \pm 0.2$ & $14.6 \pm 0.3$ & $12.0 \pm 0.2$ \\
-0.6 to -0.1 & $14.5 \pm 0.3$ & $11.9 \pm 0.2$ & $6.5 \pm 0.3$ & $23.7 \pm 0.5$ & $13.2 \pm 0.4$ \\
-0.1 to 0.6 & $24.1 \pm 0.6$ & $13.3 \pm 0.4$ & & \\
\hline
\end{tabular}

In order to minimize the contamination from the halo, we only consider stars with rotational velocity $V_{\phi}>50 \mathrm{~km}$ $\mathrm{s}^{-1}$, which is consistent with Sales et al. (2009). Our sample also have eliminated the small fraction of stars with metallicity $[\mathrm{Fe} / \mathrm{H}]<-1.2$ (Carollo et al. (2010) shows these stars may be chemically attributable to the halo). We then examine the eccentricity distribution of stars in different range of metallicities and heights from the Galactic plane to estimate the contamination of the thin-disk, as the thin-disk stars dominate in close distance and metal-rich. The result is shown in Figure 10. It shows that these eccentricities distributions in different $|Z|$ and $[\mathrm{Fe} / \mathrm{H}]$ ranges look similar, with a peak at low eccentricity and a pronounced asymmetric tail toward high eccentricities $(e \sim 0.8)$. The thick-disk should dominate at larger distance $(|\mathrm{Z}|>1 \mathrm{kpc})$ from Galactic plane. Comparison of the three panels in Figure 10 shows that the relative frequency slightly decreases for the high eccentricity and increase for the low eccentricity at smaller $|Z|$ distance where the thin-disk stars should dominate. Considering more metal-rich stars or more contamination from thin-disk, the same trend of the relative frequency exists. We note that these changes are not significant, and it is robust that eccentricity distribution of thick-disk stars has a peak at $e \sim 0.2$ and exhibits extended tails of higher eccentricities up to $e \sim 0.8$.

We compare the result with the four published models predictions in Sales et al. (2009), as shown in Figure 11, the four models are accretion model adopted from Abadi et al. (2003), heating model from Villalobos \& Helmi (2008), radial migration model from Roškar et al. (2008a), and the gas-rich merger model from Brook et al. (2004, 2005), respectively. The observed thick-disk stars are selected at $0.8<|\mathrm{Z}|<2.4 \mathrm{kpc}$ (taking the scale height $\mathrm{Z}_{0}$ of the thick disk as $0.8 \mathrm{kpc}$ ) consisting with the range $1<\left|\mathrm{Z} / \mathrm{Z}_{0}\right|<3$ in the Sales et al. (2009), $-1.2<[\mathrm{Fe} / \mathrm{H}]<-0.6$ and also $V_{\phi}>$
$50 \mathrm{~km} \mathrm{~s}^{-1}$. For the accretion model, the distribution is symmetric, very broad and having a median eccentricity $e \sim 0.5$, which is not consistent with our distribution. Although the distributions from heating model has a peak at low eccentricity, it also has secondary peak at high eccentricity which does not have in our distribution. Figure 3 of Sales et al. (2009) shows this secondary peak is mostly occupied by the accreted stars (which retain the initial orbital characteristics of the merging satellite), and Di Matteo et al. (2011) found that fewer stars with extreme values and no evidence of their secondary peak around $e \sim 0.8$ in their simulation with the small satellite mass (1:10 mass ratio). Di Matteo et al. (2011) also found that the increase of the stellar orbital eccentricities in the solar neighborhood with vertical distance, which can also be found from our Figure 10, can be reproduced if the satellite is accreted onto a direct orbit. For the migration model, the distribution is more Gaussian like, exhibiting a symmetry about the peak until it gets down to the high-e tail. But it is not displayed in our distribution, making this particular realization of the migration model somewhat less consistent with our result. Our result is consistent with the distribution from gas-rich merger model which exhibits an asymmetric peak.

Some studies compared the above models expectations with observed distributions of orbital eccentricities for thickdisk stars in the solar neighborhood, e.g. Dierickx et al. (2010), Wilson et al. (2011), Lee et al. (2011). Based on different samples, these studies adopt different models of the Milky Way potential, and all produce similar eccentricity distributions for the thick-disk stars, namely, a peak at low eccentricity and extended a tail to higher eccentricity. Considering above studies about stellar orbital eccentricities, the favored mechanisms for thick-disk formation are likely to be either (or both) the gas-rich mergers model or the thin-disk heating by minor mergers scenario. 


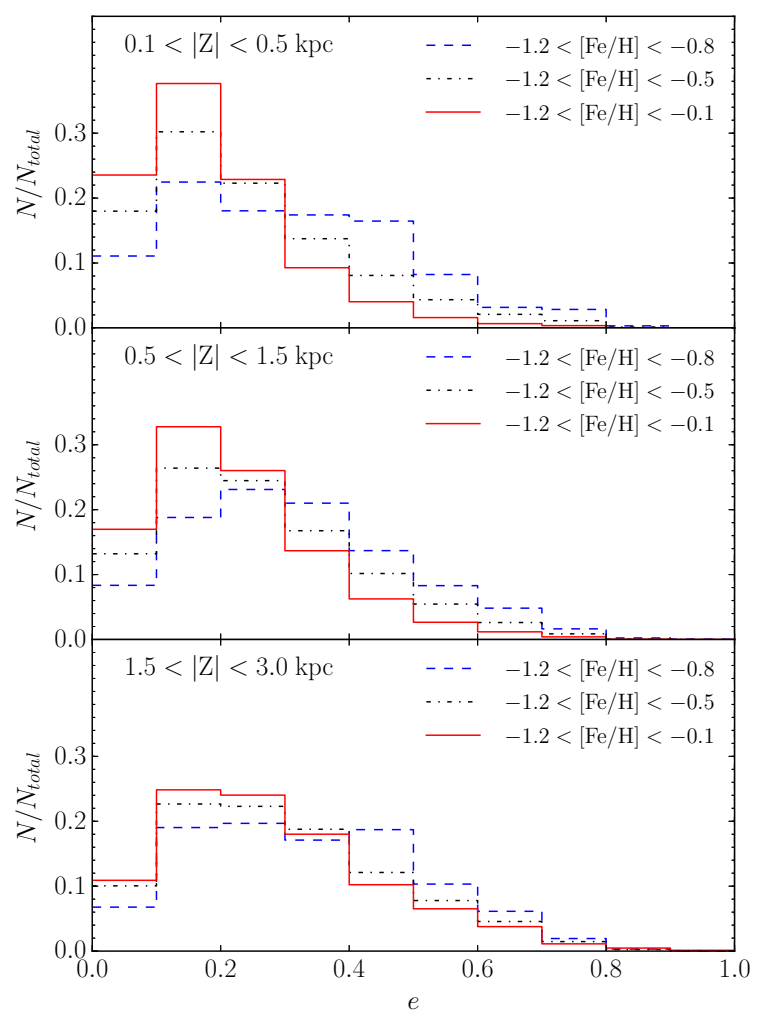

Figure 10. Eccentricities distributions for different $|Z|$ and metallicities $[\mathrm{Fe} / \mathrm{H}]$.

\section{SUMMARY AND CONCLUSIONS}

In this paper, we use $130,043 \mathrm{~F} / \mathrm{G}$-type dwarf stars from the LAMOST DR2 data to investigate kinematics and metallicity distribution of the Galactic disk. Our sample comprises stars with $6.5<R<9.5 \mathrm{kpc}, 0.1<|Z|<3 \mathrm{kpc}, \log g>3.5$, $-1.2<[\mathrm{Fe} / \mathrm{H}]<0.6$, and $\mathrm{S} / \mathrm{N}>15$. It shows that our sample stars could be mainly contributed from the disk system.

In the intermediate-metallicity range $-1.2<[\mathrm{Fe} / \mathrm{H}]$ $<-0.5$, the orbital eccentricity generally decreases with increased metallicity, and there is a relatively larger gradient. In the metal-rich range $-0.2<[\mathrm{Fe} / \mathrm{H}]<0.6$, there is little or no correlation between orbital eccentricity and metallicity $[\mathrm{Fe} / \mathrm{H}]$ for stars at $0.1<|Z|<0.5 \mathrm{kpc}$ and $0.5<|Z|<1.0$ $\mathrm{kpc}$, however, for stars farther from the Galactic plane $(1.0<|Z|<3.0 \mathrm{kpc})$, the eccentricity has a little increase with increasing metallicity. Those trends are also found in the correlation between $\sigma_{U}$ and $\sigma_{V}$ with metallicity. Moreover, the observed thick-disk stellar orbital eccentricity distribution peaks at low eccentricity $(e \sim 0.2)$ and extends to a high eccentricity $(e \sim 0.8)$. We then compare this result with four thick-disk formation models, and it appears that the observed distribution is consistent with the gas-rich merger model and against with accretion model.

We examine the rotational velocity with $|Z|$ and $R$ at the high-metallicity $([\mathrm{Fe} / \mathrm{H}]>-0.1)$ and intermediate-metallicity range $(-0.8<[\mathrm{Fe} / \mathrm{H}]<-0.6)$. It show that there exists a clear gradient of $V_{\phi}$ with $|Z|$, and there is only a negligible rotational velocity gradient with the Galactocentric radius $R$ for both metallicity range. In addition, the rotation veloc-

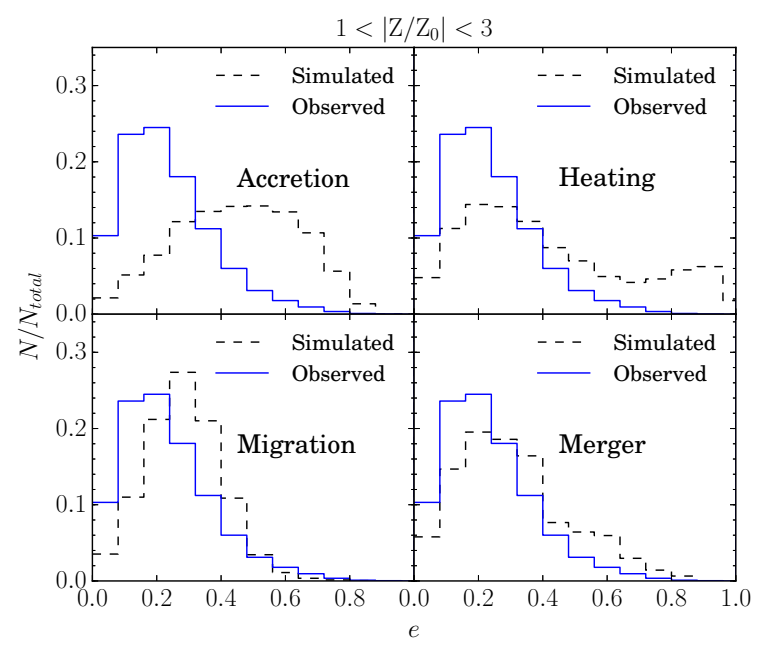

Figure 11. Eccentricities distributions for our observed thickdisk stars and model predictions from Sales et al. (2009). The observed data are restricted at $0.8<|\mathrm{Z}|<2.4 \mathrm{kpc},-1.2<[\mathrm{Fe} / \mathrm{H}]$ $<-0.6$ and $V_{\phi}>50 \mathrm{~km} \mathrm{~s}^{-1}$.

ity increases with increased metallicity $[\mathrm{Fe} / \mathrm{H}]$ in the range $-1.2<\mathrm{Fe} / \mathrm{H}]<-0.2$ and has a peak at $[\mathrm{Fe} / \mathrm{H}] \sim-0.2$, then it becomes a slightly decrease when $[\mathrm{Fe} / \mathrm{H}]>-0.2$. After separating the sample stars into likely thin-disk and thick-disk sub-sample, we find that there exits a negative gradient of rotation velocity $V_{\phi}$ with metallicity $[\mathrm{Fe} / \mathrm{H}]$ for the likely thin-disk sub-sample, and the thick-disk sub-sample exhibit a larger positive gradient of rotation velocity with metallicity. The gradient for the likely thin-disk sub-sample qualitatively agrees with the predictions of the radial migration models (Loebman et al. 2011). We consider the radial migration of stars appears to have influenced on the thin-disk formation. The gradient for the thick-disk sub-sample is consistent with the result of pure N-body models of Curir et al. (2012). However, to detailed quantitative comparisons with these observation results, it need to constructed more physically realistic models and simulations.

\section{ACKNOWLEDGEMENTS}

We especially thank the referee for his/her insightful comments and suggestions which have improved the paper significantly. This work was supported by joint fund of Astronomy of the National Natural Science Foundation of China and the Chinese Academy of Science, under Grants U1231113. This work was also by supported by the Special funds of cooperation between the Institute and the University of the Chinese Academy of Sciences. In addition, this work was supported by the National Natural Foundation of China (NSFC, No.11373033, No.11373035), and by the National Basic Research Program of China (973 Program) (No. 2014CB845702, No.2014CB845704, No.2013CB834902). Guoshoujing Telescope (the Large Sky Area Multi-Object Fiber Spectroscopic Telescope LAMOST) is a National Major Scientific Project built by the Chinese Academy of Sciences. Funding for the project has been provided by the National Development and Reform Commission. LAMOST is oper- 
ated and managed by the National Astronomical Observatories, Chinese Academy of Sciences.

\section{REFERENCES}

Abadi M. G., Navarro J. F., Steinmetz M., Eke V. R., 2003, ApJ, 597,21

Aihara H., et al., 2011, ApJS, 193, 29

Beers T. C., et al., 2006, Mem. Soc. Astron. Italiana, 77, 1171

Bensby T., Feltzing S., Lundström I., 2003, A\&A, 410, 527

Bensby T., Zenn A. R., Oey M. S., Feltzing S., 2007, ApJ, 663, L13

Bensby T., Alves-Brito A., Oey M. S., Yong D., Meléndez J., 2011, ApJ, 735, L46

Bensby T., Feltzing S., Oey M. S., 2014, A\&A, 562, A71

Binney J., et al., 2014, MNRAS, 439, 1231

Bond N. A., et al., 2010, ApJ, 716, 1

Bovy J., et al., 2012, ApJ, 759, 131

Bovy J., Bird J. C., García Pérez A. E., Majewski S. R., Nidever D. L., Zasowski G., 2015, ApJ, 800, 83

Brook C. B., Kawata D., Gibson B. K., Freeman K. C., 2004, ApJ, 612, 894

Brook C. B., Gibson B. K., Martel H., Kawata D., 2005, ApJ, 630, 298

Brook C., Richard S., Kawata D., Martel H., Gibson B. K., 2007, ApJ, 658, 60

Carollo D., et al., 2010, ApJ, 712, 692

Casetti-Dinescu D. I., Girard T. M., Korchagin V. I., van Altena W. F., 2011, ApJ, 728, 7

Cheng J. Y., et al., 2012, ApJ, 752, 51

Chiba M., Beers T. C., 2000, AJ, 119, 2843

Cui X.-Q., et al., 2012, RAA, 12, 1197

Curir A., Lattanzi M. G., Spagna A., Matteucci F., Murante G., Re Fiorentin P., Spitoni E., 2012, A\&A, 545, A133

Deng L.-C., et al., 2012, RAA, 12, 735

Di Matteo P., Lehnert M. D., Qu Y., van Driel W., 2011, A\&A, $525, \mathrm{~L} 3$

Dierickx M., Klement R., Rix H.-W., Liu C., 2010, ApJ, 725, L186

Du C., et al., 2003, A\&A, 407, 541

Du C., Ma J., Wu Z., Zhou X., 2006, MNRAS, 372, 1304

Eisenstein D. J., et al., 2011, AJ, 142, 72

Feast M. W., Whitelock P. A., 1997, in Hipparcos - Venice '97. pp 625-628

Freeman K. C., 1987, ARA\&A, 25, 603

Freeman K., Bland-Hawthorn J., 2002, ARA\&A, 40, 487

Fuhrmann K., 1998, A\&A, 338, 161

Fuhrmann K., 2008, MNRAS, 384, 173

Gilmore G., Reid N., 1983, MNRAS, 202, 1025

Gilmore G., Wyse R. F. G., 1985, AJ, 90, 2015

Gratton R., Carretta E., Matteucci F., Sneden C., 1996, in Formation of the Galactic Halo...Inside and Out. p. 307

Gunn J. E., Knapp G. R., Tremaine S. D., 1979, AJ, 84, 1181

Hayden M. R., et al., 2015, ApJ, 808, 132

Ivezić Ž., et al., 2008, ApJ, 684, 287

Jia Y., et al., 2014, MNRAS, 441, 503

Jurić M., et al., 2008, ApJ, 673, 864

Kordopatis G., et al., 2015, A\&A, 582, A122

Lee Y. S., et al., 2011, ApJ, 738, 187

Loebman S. R., Roškar R., Debattista V. P., Ivezić Ž., Quinn T. R., Wadsley J., 2011, ApJ, 737, 8

Luo A.-L., et al., 2012, RAA, 12, 1243

Majewski S. R., 1993, ARA\&A, 31, 575

Minchev I., Famaey B., 2010, ApJ, 722, 112

Miyamoto M., Nagai R., 1975, PASJ, 27, 533

Moni Bidin C., Carraro G., Méndez R. A., van Altena W. F., 2010, ApJ, 724, L122

Moni Bidin C., Carraro G., Méndez R. A., 2012, ApJ, 747, 101
Moultaka J., Ilovaisky S. A., Prugniel P., Soubiran C., 2004, PASP, 116, 693

Munn J. A., et al., 2004, AJ, 127, 3034

Munn J. A., et al., 2008, AJ, 136, 895

Ness M., et al., 2016, ApJ, 819, 2

Ojha D. K., Bienayme O., Robin A. C., Creze M., Mohan V., 1996, A\&A, 311, 456

Paczynski B., 1990, ApJ, 348, 485

Pompéia L., Barbuy B., Grenon M., 2002, ApJ, 566, 845

Prochaska J. X., Naumov S. O., Carney B. W., McWilliam A., Wolfe A. M., 2000, AJ, 120, 2513

Quinn P. J., Goodman J., 1986, ApJ, 309, 472

Recio-Blanco A., et al., 2014, A\&A, 567, A5

Reddy B. E., Lambert D. L., Allende Prieto C., 2006, MNRAS, 367, 1329

Roškar R., Debattista V. P., Stinson G. S., Quinn T. R., Kaufmann T., Wadsley J., 2008a, ApJ, 675, L65

Roškar R., Debattista V. P., Quinn T. R., Stinson G. S., Wadsley J., 2008b, ApJ, 684, L79

Sales L. V., et al., 2009, MNRAS, 400, L61

Schönrich R., Binney J., 2009, MNRAS, 396, 203

Schönrich R., Binney J., Dehnen W., 2010, MNRAS, 403, 1829

Sellwood J. A., Binney J. J., 2002, MNRAS, 336, 785

Siebert A., et al., 2008, MNRAS, 391, 793

Siebert A., et al., 2011, AJ, 141, 187

Smith M. C., et al., 2009, MNRAS, 399, 1223

Spagna A., Lattanzi M. G., Re Fiorentin P., Smart R. L., 2010, A\&A, 510, L4

Spitoni E., Matteucci F., 2011, A\&A, 531, A72

Su D.-Q., Cui X.-Q., 2004, Chinese J. Astron. Astrophys., 4, 1

Tian H.-J., et al., 2015, ApJ, 809, 145

Villalobos Á., Helmi A., 2008, MNRAS, 391, 1806

Villalobos Á., Kazantzidis S., Helmi A., 2010, ApJ, 718, 314

Wang S.-G., Su D.-Q., Chu Y.-Q., Cui X., Wang Y.-N., 1996, Appl. Opt., 35, 5155

Wilson M. L., et al., 2011, MNRAS, 413, 2235

Wu Y., et al., 2011, RAA, 11, 924

Yanny B., et al., 2009, AJ, 137, 4377

York D. G., et al., 2000, AJ, 120, 1579

Zhao G., Zhao Y.-H., Chu Y.-Q., Jing Y.-P., Deng L.-C., 2012, RAA, 12,723

This paper has been typeset from a $\mathrm{TE}_{\mathrm{E}} \mathrm{X} / \mathrm{LATEX}$ file prepared by the author. 